\title{
Unfall- und Sachverständigenkunde
}

Un ter s u c hu ng e nüber A u ge n m a g n e t e. Vortrag, gehalten von S. Turk in der Berliner medizinischen Gesellschaft am 27. Juni 1900. Centralbl. f. Augenh. Juliheft 1900. Verf. hat an 8 Eisenplittern irn Gewichte von 0,001 bis 0,5 g Ver-suche über die Anziehungskraft desHaab'schen und desHirschberg'schen Magneten in verschiedenen Abständen (von $5-0 \mathrm{~cm}$ ) angestellt. Er maass die Anziehungskraft an der Belastung, die die angezogenen Splitter eben noch trugen, ohne losgerissen zu werden. Aus den Messungen ist zu entnehmen (was nach physikalischen Gesetzen auch Torauszusetzen ist), dass 1. die anziehenden Kräfte bei jedem Magneten um so geringer sind, je kleiner der anzuziehende Splitter ist : 2. dass far Splitter von 0,001 - 0,25 g Gewicht bei direkter Berührung die Kraft des H ir s ch b er g'schen Magneten relativ wenig (um 1/5-1/3) hinter der des II a a b'schen zurücksteht, während die Differenz ganz bedeutend wird - schon bei $0,2 \mathrm{~cm}$ Abstand zieht der Haab'sche Magnet das $9-23$ fache je nach der Grösse des Splitters - wenn ein Zwischenraum den Fremdkörper von dem Magneten trennt. Die Ueberlegenheit des grossen Magneten liegt also in der Fernwirkung. Da letztere sich bei der Anziehung mit zunehmender Annäherung des Splitters ganz bedeutend verstärkt - sie beträgt z. B. fur die kleinsten Splitter bei $0,5 \mathrm{~cm}$ das 266 fache, vvie bei $5^{\prime} \mathrm{cm}$, für die grossen (von 0.5 gj das 20 fache - so empfiehlt Vortragender, um für die Anziehungsstrecke innerhalb des $\mathrm{Au}^{\circ} \cdot$ es die anziehende Kraft möglichst gleichmässig zu gestalten, das gauze Operationsgebiet möglichst weit vom Magneten zu halten. Denn die Unterschiede innerhalb einer Strecke sind um so geringer, je weiter letztere vom Pol des Magneten entfernt ist. In der Diskussion zu diesemVortrage erweiterteProf. Hirschberg die Ausführungen Turk's nach der praktischen Seite. Er betonte die Wichtigkeit eines gut funktionierenden, zeitgemäss eingerichteten In-strumentariums ; in seiner Klinik habe er stets zwei Exemplare des handlicheu Magneten in Gebrauch. die Accumulatoren seien doppelt und dreifach vorhanden und stets kontrolliert, ausserdem benutzt er noch den Schlosserschen Magneten und einen nach Haab von Hirschmann in Berlin angefertigten Riesenmagneten von 80 Kilo (iewicht. Er weist bei dieser Gelegenheit die Vorwürfe, die gegen seinen kleinen Magneten erhoben $\Lambda$ vürden, sehr entschieden zurück : es sei eine Fabel, dass er keine Fernwirkung habe, es sei eine $\Lambda$ veitere Fabel, dass vorsichtige Einführung des aseptischen Magnetendes in den Glaskörper Netzhautablösung bewirke. Er hebt ferner hervor, dass sein verbessertes Side-roscop bei elektrischem Strassenbahnbetrieb brauchbarer sei, als dasim selben Raum aufgestellte Asmus'sche (auch ebenso empfindlich für Lokalisation sehr kleiner Splitter ? I). Rf.) Für die Magnetoperationen stellt Herr Hirschberg folgende Leitsätze auf: 1. In ganz frischen

162

Therapeutische Umschau.

Fallen ist der kleine Magnet nicht bios bequem anwendbar, sondern demmittleren überlegen, während der Riesenmagnet direkt gefährlich ist unddeshalb auf das noch offene Auge nicht angewendet werdensoil; 2. ist die Wunde geschlossen. aber der Splitter noch nicht festangewachsen, so lockt man ihn mit Hilfe des mittelgrossen Magneten indie Vorderkammer, macht Schnitt am untern Hornhautrand und extrahirtmit dem kleinen Magneten ; 3. ist der 
Splitter fest eingewachsen, sogelingt Lockerung mit dem mittleren Magneten nur, wenn der Splitternicht zu klein ist, 20 - 30 mgr wiegt. Da ist dann der Riesenmagnetam Platze. Sind jedoch die Splitter sehr klein, unter 3 mgr, so ver-sagt auch er moistens und Schnitt und kleiner Magnet kouimen $\Lambda$ viederan die Reihe. Ist die Linse getrübt, und sitzt der Fremdkörper inivorderen Teil der Netzhaut, so macht Hirschberg eine breite Iridectomiein der Richtung des Fremdkörpers und geht mit seinem kleinen Magnetenzwischen Linse und Augapfel vor. Sonst kommt vorderer oder hintererMeridionalschnitt in Frage. Pfalz.

Therapeutische Umschau.

Ueber subconjunctivale Einspritzungen mit Acoin. Dr. G. Hirsch.

Halberstadt. (Archiv f. Augenheilkunde, November 1900.)

Verf. bedauert es, dass über das vor Jahresfrist von Darier zur Beseitigung des Schmerzes bei subconjunctivalen Injectionen empfohlene Acoin keine weiteren Erfahrungen mitgetheilt sind Nach eigenen Versuchen Hirsch's lässt das Acoin Pupille, Accommodation sowie Gefässfüllung der Bindehaut unbeeinflusst, und \rergiftungserscheinımgen werden niemals beobachtet. Die Anästhesie, welche das Mittel, in 1 proc. Lösung tropfenweise applicirt, auf Cornea und Bindehaut in physiologischem und gereiztem Zustande hervorbringt, ist eine vollkommene und länger andauernde als die nach Cocain, nur muss man 2-5 Minuten nach der Eintröpfelung warten, bis die voile Wirkung eingetreten ist.

Besonders versucht und bewährter befunden als Cocain wurde das Anästheticum bei subconjunctivalen Injectionen an Hydrarg. cyanatum, Kochsalz etc.

Wenn man kurz vor dem Einstich in die Conjunctiva diese mit Acoinlösung betropft und Acoin auch der zur Injection bestimmten Lösung hinzufügt, so erreicht man es, dass die Patienten sich den - sonst bekanntlich sehr schmerzhaften - Einspritzungen willig unter-ziehen und die $\Lambda$ Yiederholung derselben ohne Schwierigkeiten gestatten.

Einige Fälle, $\Lambda$ velche den therapeutischen Werth der subconjunctivalen Injectionen an sich illustriren sollen, $\Lambda$ verden angefiihrt.

Ueber die Bedeutung der Luftinfection fur das Auge. Dr. S. Lobanoff, St. Petersburg. (Von Gräfe's Archiv, Band LI, 3. Heft.

L. Lobanoff hat im Laboratorium der Rostocker Universitäts-Augenklinik experimentelle Untersuchungen über die Bedeutung der Luft- 